\title{
Pigmentation in the oral cavity - a report of a rare case of malignant melanoma
}

\section{Introduction}

Melanoma is a malignant neoplasm of melanocytic origin that arises from a benign melanocytic lesion. ${ }^{1}$ In 1855, Oral malignant melanoma (OMM) was first coined the term by Weber. ${ }^{1,2}$ Malignant melanoma of oral cavity found in the basal layer of the oral mucous membranes which is extremely rare tumor arising from the uncontrolled growth of melanocytes. The World Health Organization (WHO) has defined mucosal malignant melanoma as a malignant neoplasm of melanocytes or of melanocyte predecessors. ${ }^{1,2}$ It is characterized by the proliferation of atypical melanocytes at the epithelial-connective tissue interface, associated with upward migration into the epithelium and by invasion of the underlying connective tissues. ${ }^{2,3}$ Only about $1 \%$ of all melanomas arise in the oral mucosa and these accounts for $0.5 \%$ of all oral malignancies. ${ }^{4}$ These rare lesions may present themselves in yet uncommon ways which need to be identified and reported to understand their biologic behavior, and hence this case report is an attempt do the same.

\section{Case report}

A 65 year old male patient sabjibhai solanki reported to the department of oral medicine and radiology with chief complain of pain and swelling in upper right back tooth region since one months, started as pin point lesion, progressed gradually to attend present size and shape. On clinical examination, a solitary diffuse swelling was present on the right posterior region maxillary teeth region i.r.t. 1618 , measuring about $3 \times 2 \mathrm{~cm}$ oval in shape, extending from distal 15 to about $1 \mathrm{~cm}$ before the maxillary tuberosity. The overlying mucosa was dark blue in color and without any associated secondary changes. There was multiple area of brown pigmentation having variable sizes ranging from pin point to about $1 \mathrm{~mm}$ in greatest dimension throughout the soft palate area (Figure 1). On palpation the lesion was smooth, non-tender, well defined, firm to hard in consistency with induration of the posterior margins. General physical examination was insignificant and vital signs within normal limits. Extra oral examination revealed solitary right sub mandibular lymph node was palpable; a solitary cervical lymph node was also palpable on right side which was mobile, non-tender and firm.

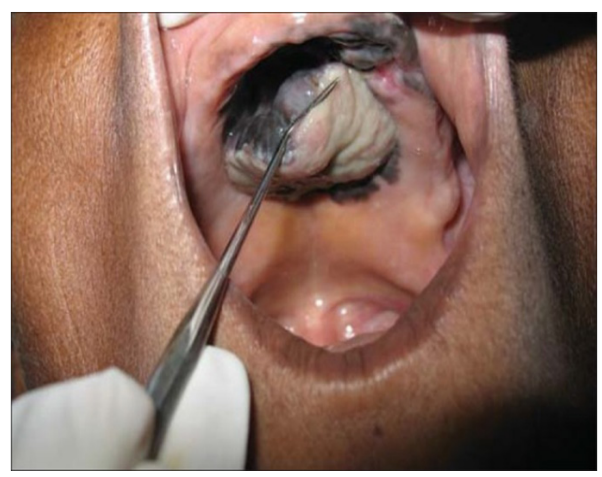

Figure I Clinical picture showing a pedunculated lesion involving the palate.
Volume 9 Issue 3 - 2018

\author{
Parthsarthi Gautam \\ Narsinhbhai Patel Dental College and hospital, India
}

Correspondence: Parthsarthi Gautam, Narsinhbhai Patel Dental College and hospital, India, Tel 8340210730, Email parthsarthi.gautam@gmail.com

Received:June 12, 2017 | Published: May 16, 2018

Pigmented nodular growth was present with color of overlying mucosa was bluish brown extending from edentulous portion of 15 -18 , oval in shape, obliterating right buccal vestibule. Based on clinical appearance, the provisional diagnosis was malignant melanoma while pigmented lesions were like Hemangioma, AV malformation and melanoma were considered under differential diagnosis. Blood investigations did not reveal any significant findings but radiographic features revealed ill-defined radiopaque area involving posterior right alveolus region posterior to 15 extending up to maxillary tuberosity with ragged border. Incisional biopsy of the lesion was performed and sent for histopathological examination. Haematoxylin and eosin-stained sections showed Para keratinized stratified squamous epithelium and connective tissue stroma. Connective tissue stroma is comprised of sheets and island of neoplastic cells. Lesional cells consisted of round to polygonal and spindle shape cells. Anisocytosis and anisonucleosis along with increased number of mitotic figures were observed. Neoplastic cells consisted of hyperchromatic nucleus. One area of the section shows melanin pigmentation in the connective tissue stroma and also in the cytoplasm of the neoplastic cells. The lesion was diagnosed as melanoma. Further this was confirmed. Malignant melanoma is a rare aggressive neoplasm, which represents $1-2 \%$ of oral malignancies.

\section{Discussion}

Malignant melanoma is a neoplasm of epidermal melanocytes and is the $3 \mathrm{rd}$ most common cancer of skin and $3 \%$ of all such malignancies. Incidence among dark skinned ethinic groups is 1 per lakh or less and in light skinned caucasians up to $50 \%$ or high. ${ }^{5}$ Sunlight is an important etiologic factor in cutaneous melanoma some melanomas developed in preexisting pigmented nevi particularly junctional nevi. Certain lesions are considered to be the precursor lesions of melanoma including acquired nevi, dysplastic nevus, congenital nevus and cellular blue nevus. Other environmental etiological factors are artificial U.V source; socioeconomic status, fair skin freckles, and number of melanocytic nevi. ${ }^{6-8}$ Genetic factors are familial melanoma and xeroderma pigmentosum. Oral mucosal melanomas have no apparent relationship to chemical thermal or physical agents. Benign intra oral melanocytic proliferations (nevi) occur and are potential sources of some oral melanomas. Currently most oral melanomas are thought to arise de-novo. ${ }^{6-8}$ Most melanomas are seen in white 
adults. Average age of affected persons is 50 to 55 years but they are evenly distributed over 30 to 80 year age. Males are more commonly affected. ${ }^{9}$ malignant cells often nest in groups in an organoid fashion however single cells can predominate Melanoma cells have large nuclei often with prominent nucleoli. They show nuclear pseudo inclusions due to nuclear membrane irregularities Oral lesions tend to show invasion of lymphatic and blood vessels more readily than skin lesions Immunohistochemical studies showing S-100 protein, MART -1 and HMB -45 reactivity of the lesional cells are beneficial in distinguishing such melanomas from other malignancies. ${ }^{10,11}$ The treatment of oral melanoma still is surgical excision. Jaw resection and lymph node dissection is indicated in cases involving bone and regional lymph nodes. Surgical margins of 3 to $5 \mathrm{~cm}$ around the tumor are necessary to achieve control. Recent studies indicate that a $1 \mathrm{~cm}$ margin is adequate for small early tumors. ${ }^{13}$ Other forms of therapy such as cryosurgery, radiation, chemotherapy and immunotherapy have been employed. Oral mucosal melanomas have a far worse prognosis than cutaneous melanomas. ${ }^{14}$ Histological features which are of prognostic significance are histologic type and depth of invasion. The level of tumor invasion is another important indicator for the prognosis of malignant melanoma. ${ }^{13}$ CLARK system is generally used to grade tumor invasion based on the deepest histologic cutaneous structure the tumor infiltrates. ${ }^{13}$

\section{Conclusion}

Although oral malignant melanomas are rare with poor prognosis, proper diagnosis and appropriate treatment modalities may increase the survival rate of the patient. Melanomas in the palate and the maxillary alveolar ridge may sometimes present as a polypoid mass, however the presentation as a pedunculated mass in extremely rare, with only a few reported cases, as present in this case. The unusual clinic-pathologic presentation of the case and the conflicting biologic behavior makes it essential that such cases be reported and studied further to evaluate their long term behavior and prognosis.

\section{Acknowledgements}

None.

\section{Conflict of interest}

The author declares that there is no conflict of interest.

\section{References}

1. Ullah H, Vahiker S, Singh M, et al. Primary malignant mucosal melanoma of the oral cavity: A case report. Egypt J Ear Nose Throat Allied Sci. 2010;11:48-50.

2. Dimitrakopoulos I, Lazaridis N, Skordalaki A. Primary malignant melanoma of the oral cavity. Report of an unusual case. Aust Dent J. $1998 ; 43: 379-81$.

3. Pour MS. Malignant melanoma of oral cavity. J Dent. 2007;4:44-51.

4. Speight PM. Lyon: IARC Press; 2005. Mucosal malignant melanoma. World Health Organization Classification of Tumours, Pathology and Genetics of Head and Neck Tumours; pp. 206-7.

5. Vikey AK, Vikey D. Primary malignant melanoma, of head and neck: A comprehensive review of literature. Oral Oncol. 2012;48(5):399-403.

6. Gondivkar SM, Indurkar A, Degwekar S, et al. Primary oral malignant melanoma: A case report and review of the literature. Quintessence Int. 2009;40(1):41-6.

7. Rajendran R, Sivapathasundharam B. Shafer, Hine, Levy's Shafer's Text Book of Oral Pathology. 6th ed. Vol. 80. India: Elsevier; Benign andmalignant tumors of the oral cavity; 2009: 218.

8. Manganaro A, Hammond H, Dalton M, et al. Oral melanoma: Case report and review of literature. Oral Surg Oral Med Oral Pathol Oral Radiol Endod. 1995;80(6):670-6.

9. Hicks MJ, Flaitz CM. Oral mucosal melanoma: Epidemiology and pathobiology. Oral Oncol. 2000;36(2):152-69.

10. Strauss JE, Strauss SI. Oral malignant melanoma: A case report and review of literature. J Oral Maxillofac Surg. 1994;52:972-6.

11. Devi P, Bhovi T, Jayaram RR, et al. Malignant melanoma of the oral cavity showing satellitism. J Oral Sci. 2011;53(2):239-44.

12. Pfister DG, Ang KK, Brizel DM, et al. Head and neck cancers, version 2.2013. Featured updates to the NCCN guidelines. J Natl Compr Canc Netw. 2013;11(8):917-923.

13. Kingdom TT, Kaplan MJ. Mucosal melanoma of the nasal cavity and paranasal sinuses. Head Neck. 1995;17(3):184-189. 